\title{
Divorcing Jack et An Everlasting Piece : les comédies à l'écran et le processus de paix en Irlande du Nord
}

\section{Cécile Bazin}

\section{(2) OpenEdition \\ Journals}

Édition électronique

URL : http://journals.openedition.org/etudesirlandaises/2386

DOI : $10.4000 /$ etudesirlandaises. 2386

ISSN : 2259-8863

Éditeur

Presses universitaires de Rennes

\section{Édition imprimée}

Date de publication : 30 décembre 2011

Pagination : 87-102

ISSN : 0183-973X

\section{Référence électronique}

Cécile Bazin, « Divorcing Jack et An Everlasting Piece : les comédies à l'écran et le processus de paix en Irlande du Nord », Études irlandaises [En ligne], 36-2 | 2011, mis en ligne le 30 septembre 2013, consulté le 19 avril 2019. URL : http://journals.openedition.org/etudesirlandaises/2386 ; DOI :

10.4000/etudesirlandaises.2386

Ce document a été généré automatiquement le 19 avril 2019.

(c) Presses universitaires de Rennes 


\title{
Divorcing Jack et An Everlasting Piece : les comédies à l'écran et le processus de paix en Irlande du Nord
}

\author{
Cécile Bazin
}

1 Divorcing Jack (1998) de David Caffrey et An Everlasting Piece (2000) de Barry Levinson sont deux films qui explorent le conflit politique nord-irlandais, particulièrement la période du processus de paix. Cet article s'articule autour du rôle (et de la capacité) des comédies en Irlande du Nord pour aborder les traumatismes du passé et laisser entrevoir un espoir de paix. Le cinéma, dans sa construction discursive, à travers sa voix indépendante et sa portée populaire, offre un véhicule unique pour l'exploration des Troubles et du processus de paix. La comédie, qui est un genre nouveau parmi les films traitant ce sujet, permet quant à elle de transgresser les tabous de la violence politique en Irlande du Nord, et porte à l'écran de manière inédite le processus de paix. On prendra ici en compte le dialogue entre le cinéma traitant du conflit nord-irlandais et la société concernée, et l'on sera amené à s'interroger sur l'incidence du processus de paix en Irlande du Nord sur les représentations cinématographiques du conflit et inversement. Notons par ailleurs que la plupart des films traitant à la fois des Troubles et du processus de paix ont été réalisés au cours des années 1990 et 2000. C'est à partir de la signature de l'Accord du Vendredi Saint (avril 1998) que les comédies sur le conflit politique nord-irlandais sont réalisées. Il s'agira ici de démontrer que Divorcing Jack (1998) et An Everlasting Piece (2000) fournissent un nouvel espace - ancré dans la comédie pour aborder le processus de paix en Irlande du Nord, au-delà du contexte politique. En effet, ces deux films reflètent particulièrement la mouvance du processus de paix, dans la mesure où ils remettent en question la violence politique en la ridiculisant. Il s'agit de deux comédies politiques qui revisitent les Troubles et leurs conséquences à la lumière du processus de paix et révèlent que lorsque la violence politique s'achève, il devient possible d'explorer le conflit en Irlande du Nord. Contrairement aux films réalisés pendant les Troubles ${ }^{1}$, qui représentaient ce conflit en Angleterre en observant principalement l'IRA, les films réalisés pendant le processus de 
paix se focalisent sur l'Irlande du Nord ${ }^{2}$. Si le renoncement à la violence politique est le thème développé dans les films réalisés pendant le processus de paix, Divorcing Jack et An Everlasting Piece témoignent également d'une reconstruction de l'Irlande du Nord en excluant, en partie, la violence paramilitaire destructrice ${ }^{3}$ et en donnant à voir de nouvelles images de Belfast. Ces dernières abordent la réconciliation entre catholiques et protestants (comme dans An Everlasting Piece, qui est centré sur le commerce aussi incongru qu'œcuménique d'un protestant et d'un catholique), et se concentrent sur l'iconographie de la ville à travers la comédie.

2 Ce faisant, ces films proposent une relecture de l'histoire, que Marc Ferro ${ }^{4}$ répertorie en quatre groupes: l'histoire officielle, l'histoire mémoire, l'histoire expérimentale et l'histoire fiction, observant que dans le cas de l'histoire fiction «on choisit des informations qui semblent significatives au moment où l'œuvre s'accomplit. Ce n'est pas le passé qui est aux commandes, comme dans l'Histoire-mémoire, mais le présent ${ }^{5} »$. Or, puisque l'histoire-fiction attribue une fonction majeure au présent, on tentera de démontrer comment, à travers la comédie, Divorcing Jack et An Everlasting Piece remettent en question la lecture habituelle des Troubles et proposent une nouvelle lecture des événements passés. On s'intéressera à la manière dont ces films représentent le processus de paix en Irlande du Nord, tout en observant ce que la fonction de comédie ajoute au dialogue entre le cinéma et le processus de paix. Au travers de ces deux films, nous montrerons comment la comédie (dont le défi, dans ce contexte, est bien de susciter le rire) permet de prendre du champ par rapport aux Troubles et invite à imaginer un avenir où la paix serait possible.

3 Par rapport aux autres films sur l'Irlande du Nord, Divorcing Jack (1998), le premier long métrage du réalisateur David Caffrey, présente la particularité de pouvoir être considéré comme un film indigène (nord-irlandais). Tout comme An Everlasting Piece, Divorcing Jack fut tourné intégralement en Irlande du Nord, alors que les autres films abordant le même sujet furent pour la plupart tournés dans le Sud ${ }^{6}$. Le film est par ailleurs issu d'une production nord-irlandaise stricto sensu, les maisons de production étant la BBC Films, Scala, The Arts Council of England et The Arts Council of Northern Ireland. Notons encore que le scénario fut écrit par Colin Bateman ${ }^{7}$, qui a lui-même adapté son roman pour le cinéma. Il convient aussi de remarquer que si les romans de Colin Bateman sont très appréciés en Irlande du Nord, ils ne connaissent en général pas le même succès de l'autre côté de la frontière. Dans un entretien avec le journaliste Mick Heaney, Colin Bateman explique qu'il a rencontré des difficultés auprès des éditeurs pour publier Divorcing Jack dans le Sud : " [...] they sent me a note saying there was no market for "a unionist thriller". It was as if they thought I put my Union Jack on in the morning before I start work ${ }^{8}$." Issu de la communauté protestante et unioniste nord-irlandaise, Bateman explique qu'il n'est pourtant en aucun cas politisé, ni religieux ${ }^{9}$. De fait, même si le film Divorcing Jack place le conflit politique au cœur du récit, les Troubles sont utilisés comme source de parodie plutôt que comme vecteur d'une vision partisane. Divorcing Jack offre une lecture satirique de la société nord-irlandaise et de sa situation politique après les Troubles ainsi que des paramilitaires loyalistes et des membres de l'IRA. Colin Bateman explique en effet que l'utilisation de ce ton insolent dans ses œuvres émane en fait de la culture punk de laquelle il se réclame : "[...] It's anti-Establishment or anti-authoritarian sarcasm. I'm not going to stand up and start a revolution but I don't have the same respect for authority other people have. I think the whole punk generation feels like that ${ }^{10}$." 
Ce film apporte un nouveau regard et apporte une vision interne sur la vie politique et la violence politique en Irlande du Nord dans la mesure où il s'intéresse aux incidences du processus de paix en Irlande du Nord et à la reconstruction de Belfast. Toutefois, malgré l'optimisme qui émane du processus de paix, Divorcing Jack en fait un portrait plutôt sombre. La période traitée représente l'Irlande du Nord à un moment que l'on peut situer juste après la signature de l'Accord du Vendredi Saint, soit en 1999 : le film explore de ce fait un sujet tout à fait unique dans l'ensemble des représentations cinématographiques de cette période, car il se projette dans l'avenir d'une Irlande du Nord constitutionnellement indépendante ${ }^{11}$, saisie au moment de la course à l'élection de son Premier ministre. Le réalisateur décrit son film comme « un mélange des genres »: «[...] $a$ mixture of genres [...] you've got that domestic break up at the start [...] then you're into the thriller genre, then you're into the political, Nixon, JFK conspiracy kind of movie [... $]^{12} »$, mais Divorcing Jack dépeint essentiellement la situation politique en Irlande du Nord et a recours ce faisant à l'humour noir. Par exemple, les meurtres que le film décrit sont perpétrés sur des victimes innocentes, mais découlent souvent de situations cocasses. Divorcing Jack relève également $\mathrm{du}$ film policier et par sa tonalité ironique, la violence paramilitaire est subvertie et parodiée. C'est notamment cette rupture de ton qui permet de qualifier Divorcing Jack de comédie politique, comme le souligne un critique: "The humour is so dangerously on the edge - like making jokes about the Holocaust - that at times you're almost afraid to laugh ${ }^{13}$.» La spécificité de Divorcing Jack se situe donc dans l'imbrication du drame et du rire, de la tragédie et de la plaisanterie. Comme Pierre Sorlin l'observe, certains films font de l'ironie « un moyen de ne pas céder au désespoir : il faut se moquer de tout, sinon la situation deviendrait intenable ${ }^{14} »$. Il note plus loin que dans les comédies de ce genre, «l'effet comique intervient de manière inattendue, souvent au terme d'une scène où s'est amorcée une fausse piste, et il rassure le spectateur qui y retrouve des situations ou des mimiques familières. Les moments dramatiques sont en revanche annoncés puis largement développés ${ }^{15}$ ». Quand bien même certains critiques jugent le ton du film irrévérencieux eu égard la complexité du conflit politique nordirlandais et aux tragédies qu'il a engendrées, pointant «[...] an irreverent and challenging black humour ${ }^{16}$ ", il faut admettre que le fait même qu'il soit désormais possible d'utiliser l'humour (qu'on le considère déplacé ou non) pour dépeindre l'Irlande du Nord témoigne d'une évolution de la situation politique et des mentalités. En raillant les hommes politiques qui seront peut-être amenés à diriger la province, et à travers eux, l'avenir institutionnel de la province, la vision de l'Irlande du Nord en devenir dans le film apparait ici quelque peu cynique. C'est d'ailleurs ce cynisme qu'incarne Dan Starkey (David Thewlis), héros malgré lui (voire anti-héros) de Divorcing Jack, qui est piégé dans une intrigue où il se trouve à la fois poursuivi par l'IRA, l'UVF et la police, et n'échappe que de peu à la mort.

5 Dan Starkey, journaliste alcoolique qui mène une vie dissolue ${ }^{17}$, écrit des colonnes satiriques sur la vie politique nord-irlandaise pour un journal de Belfast, le Belfast Evening News. Le début du film, qui reflète cette tonalité acerbe, situe l'action à la veille des élections du Premier ministre nord-irlandais. L'une des premières scènes s'ouvre sur un lâcher de ballons devant l'hôtel de ville de Belfast, et tandis que la bande sonore fait retentir les slogans qui scandent le nom de Michael Brinn, le candidat favori, le sentiment d'euphorie est tangible : «The future is bright, the future is Brinn! Vote Michael Brinn!» La journée doit être «joyeuse et optimiste» comme le patron de Dan Starkey lui demande d'écrire dans le journal, mais dans son article, le journaliste ne suit cette consigne et 
raille le potentiel futur Premier ministre en faisant allusion à ses liens avec les paramilitaires : "If elected Michael Brinn is going to swap West Belfast for the Guinness brewery in Dublin. They can have our Troubles and we can drink theirs. [...] Michael Brinn is going to sponsor paramilitary coffee mornings with an Armalite in one hand and a packet of Jaffa cakes in the other. "

Les articles de Dan Starkey révèlent donc le décalage entre l'engouement relatif pour l'indépendance de l'Irlande du Nord (incarnée par Michael Brinn) que semblent manifester les habitants et la vision cynique de cette même situation relatée par un journaliste qui ne croit pas à l'avenir supposé prometteur de l'Irlande du Nord et estime qu'il consacrerait à tout le moins un pouvoir corrompu. À travers le personnage du journaliste misanthrope, Divorcing Jack va à contre-courant de l'optimisme général, et met en scène un héros qui se marginalise et se moque de la société. Afin de renforcer le portrait satirique que fait le journaliste de la situation en Irlande du Nord, le montage insiste sur l'aspect artificiel de cette effervescence: tous les bâtiments sont filmés en surexposition et la luminosité de la ville est excessive. De même, la première prise de vue $\mathrm{du}$ candidat brosse un portrait grotesque de Michael Brinn (Robert Lindsay), car la scène de la conférence de presse, filmée en contre-plongée, souligne la démesure de la popularité du personnage. Entouré d'une horde de journalistes principalement composée de femmes, le succès du candidat doit plus à ses talents de séducteur qu'à ses talents de stratège politique éclairé, ce qui d'emblée réduit considérablement sa crédibilité. Ainsi, en suggérant que la situation politique est intrinsèquement corrompue étant donné le passé paramilitaire violent du futur Premier ministre, la perspective d'un avenir meilleur pour la ville de Belfast semble du même coup compromise et la joie ambiante surfaite. Derrière l'enthousiasme exacerbé et ces images rayonnantes et inédites célébrant la renaissance de la ville de Belfast, Divorcing Jack démontre que tout n'est qu'artifice et que cette façade ne parviendra pas à camoufler les crimes, la violence et la corruption politique qui ont profondément meurtri la ville et continueront de se perpétuer.

7 Soulignant l'absurdité de la violence en Irlande du Nord, le scénario mêle ensuite des scènes de la vie quotidienne et ordinaire à des scènes tragiques et suggère que malgré l'évolution de la situation politique et la période d'optimisme, le quotidien des Nordirlandais demeure extrêmement violent. Après être sorti acheter une pizza, Dan Starkey découvre Margaret (Laura Fraser), la jeune étudiante avec qui il a récemment eu une aventure, ensanglantée et mourante sur son lit. La jeune fille était en fait l'ancienne petite amie du leader de l'IRA, Cow Pat Keegan (Jason Isaacs). Dans l'appartement de la jeune fille, le journaliste, s'empare d'une cassette compromettante pour le politicien Michael Brinn et Cow Pat Keegan qui révèle leur affiliation à l'IRA et leur implication dans l'explosion d'une bombe qui a fait de nombreuses victimes. Dès lors Dan Starkey se trouve dans une position dangereuse, victime d'un chantage à propos de la cassette et confronté à l'enlèvement de sa propre femme par l'IRA. En outre, un autre innocent, un journaliste américain travaillant avec Dan Starkey, se fait tuer après avoir été torturé par l'IRA. Il en ressort que la paix en Irlande du Nord est utopique et relève bien de la fiction. Le film présente notamment le personnage de Michael Brinn, ancien paramilitaire candidat à l'élection du Premier ministre pour une Irlande du Nord indépendante, qui préconise la voie politique, opte pour la réconciliation et prétend mettre un terme aux activités terroristes, mais il s'avère que son discours est mensonger, puisqu'il finit par avoir luimême recours à la violence pour résoudre ses problèmes. La dernière scène du film réduit à néant tout espoir d'avenir politique en Irlande du Nord. Dan Starkey, qui détient la 
cassette compromettante, fait désormais l'objet d'un chantage moral de la part de Michael Brinn, qui veut la récupérer afin d'éviter un scandale d'Etat, et de la part de Cow Pat Keegan, qui exige en échange de l'argent et le pouvoir. On comprend à la fin de cette scène que les deux rivaux souhaitent que le journaliste relaye une image positive d'eux dans les journaux. Lorsqu'ils se retrouvent tous trois au rendez-vous pour échanger la cassette, Dan Starkey, devenu l'otage des deux terroristes, exprime son désespoir quant à la situation en Irlande du Nord. Le film finit sur l'explosion des voitures piégées par les deux hommes eux-mêmes et avec cette explosion disparaît tout candidat, toute élection et tout espoir d'Etat indépendant. Mais cette fin radicale peut néanmoins être interprétée comme un nouveau départ pour une Irlande du Nord qui resterait à construire sans criminels, sans terrorisme ni corruption politique.

Le film laisse ainsi entrevoir que la violence politique est toujours inhérente au paysage nord-irlandais mais paradoxalement, il porte à l'écran de nouvelles images de l'Irlande du Nord et plus particulièrement de la ville de Belfast, inédites dans l'ensemble des représentations cinématographiques abordant le même sujet. Et si Divorcing Jack souligne des réminiscences traumatiques liées au souvenir du passé violent des Troubles à Belfast, il s'abstient d'en faire un tableau lugubre. La ville est vivement éclairée et l'iconographie traditionnelle associée aux Troubles est absente : pas plus de tanks britanniques prenant que d'hélicoptères ou de soldats, pas de rues éventrées par les bombes ou de peintures murales, reflet du sectarisme. Le film tourné in situ explore de nouvelles représentations cinématographiques. On a ici un décor moderne de la ville qui met en scène de nouveaux quartiers :

Divorcing Jack, following the original novel [...] is [...] concerned with the depiction of Belfast and was indeed, one of the first " ceasefire " films to make use of extensive Belfast locations. For a mixture of financial and security reasons, most of the films made prior to the ceasefires substituted other locations for Belfast. Inevitably, this dependence upon stand-in locations reinforced the sense of Belfast as an abstract place of the imagination emptied of specific geographical and physical markers. Given the various cities that have substituted for Belfast (such as Dublin, London and Manchester), this has also meant that there has been little accumulation of recurring "landmarkers" [...] and little sense of Belfast as an actual lived-in space. However, by filming in the city, the makers of Divorcing Jack not only aimed to show the actuality of Belfast missing from earlier films but also to represent the 'new' Belfast emerging in the wake of the peace process. [...]Thus [...] the film also searches out locations that are new in the cinematic representation of the city: the suburbs of south Belfast, the redeveloped Cathedral Quarter and the modern concert hall and exhibition center, the Waterfront ${ }^{18}$.

9 Les édifices flambant neufs et les rues commerçantes suggérant la vie trépidante des gens de Belfast contribuent à dépeindre un tableau inédit d'une ville en voie de mondialisation et renvoient une image inédite de Belfast, ville moderne, aussi prospère que n'importe quelle autre grande ville d'Europe. De la même façon, il y a rupture dans la représentation de la police nord-irlandaise à l'image traditionnelle de la RUC, à laquelle vient se substituer une entité fictive nommée NIPD (Northern Ireland Police Department) et dont 
l'acronyme renvoie à l'image de la police new-yorkaise (NYPD : New York Police Department ). En écartant la RUC du paysage nord-irlandais, le film tente d'apaiser le conflit et de relater la paix en termes de neutralité, mais cette nouvelle image de la police à Belfast illustre également le changement généré par le processus de paix qui laisse augurer de perspectives pacifiques.

10 C'est précisément à travers la comédie que le film renouvelle la représentation de paramilitaires dont l'apparence physique des membres de l'UVF tend vers le burlesque : loin de correspondre au profil (récurrent dans les films traitant du même sujet) du psychopathe, le leader ne porte pas l'habituelle cagoule mais un chapeau, une chemise et des bottes de cow-boy. Lorsque, accompagné d'autres membres de l'UVF, il se lance à la poursuite du héros Dan Starkey (David Thewlis), c'est avec en fond sonore la musique du film Les sept mercenaires. Les paramilitaires de l'IRA ne sont pas épargnés, au point qu'en lieu et place d'une cabane délabrée au fin fond d'un comté de l'Ulster, le chef de l'IRA habite une luxueuse demeure qui a des allures de ranch. L'imagerie du western contribue ainsi à établir la dimension baroque du paysage de Divorcing Jack, où la violence paramilitaire est subvertie et parodiée et où l'excentricité des situations renforce la comédie, selon le principe que « l'excès annule la tragédie ${ }^{19}$ ».

11 Ce parti-pris satirique et burlesque remet en question tout traitement idéologique du conflit. Les paramilitaires, autant que les terroristes de tout bord, sont ridiculisés et la comédie donne à voir un tableau cocasse et caricatural des républicains et des loyalistes. L'objectif est de susciter le rire, mais aussi de créer une distance, ce qui permet de démystifier le conflit et ses protagonistes. Ce qui nous est donné à voir n'en est pas moins une vision ambivalente de l'avenir de Belfast, dans la mesure où Divorcing Jack génère certes un certain optimisme (la renaissance de la ville), mais peut-être plus encore une forme de pessimisme (véhiculée par le personnage résolument violent de Michael Brinn). En somme, le recours dans Divorcing Jack à l'humour noir fait la preuve de l'absurdité de la situation et le choix d'une iconographie inédite reflète la coexistence surréaliste à Belfast de deux univers aussi ordinaires que terrifiants, oscillant dans le sillage du processus de paix entre espérance et désespérance.

12 An Everlasting Piece, réalisé par le cinéaste américain Barry Levinson ${ }^{20}$, est quant à lui sorti en 2000. Le scénario fut écrit par Barry McEvoy, originaire de Belfast, qui interprète aussi Colm, le personnage principal du film. Malgré une bonne réception par la critique, An Everlasting Piece demeure assez méconnu du public: en dépit de la notoriété du réalisateur, de la maison de production américaine DreamWorks ${ }^{21}$ et du choix de traiter de la période des Troubles par le biais de la comédie, le film n'a pas connu le succès auquel il aurait pu prétendre. Selon le réalisateur et le scénariste, cette situation serait due à la politique de distribution de la maison de production DreamWorks qui aurait « enterré le film ${ }^{22}$ ». Le scénariste déplore que le film, qui devait être montré sur sept cent écrans ${ }^{23}$, n'ait été projeté que sur huit ${ }^{24}$ et explique cet insuccès par le fait que Barry Levinson avait refusé de couper les scènes contenant des références politiques, comme le lui avait demandé Jeffrey Katzenberg du studio DreamWorks ${ }^{25}$. Jerome O'Connor, l'un des producteurs du film qui engagea des poursuites contre la maison de production DreamWorks ${ }^{26}$, allègue que le studio avait enterré le film à la demande du gouvernement britannique $^{27}$ :

The suit, filed in Manhattan federal court, says: "the film was suppressed not because of any artistic or commercial reason, but 
rather because of political pressure" from the British government, which was unhappy over the film's mocking portrayal of British soldiers in Northern Ireland during the Troubles. The suit says that by angering the British, the film would've been an embarrassment to DreamWorks partner Steven Spielberg, who was knighted late last month and who relied on high-level British government assistance for his forthcoming World War II TV series, Band of Brothers, which was filmed in England with British army troops, military equipment and financial incentives from the British government ${ }^{28}$.

13 An Everlasting Piece représente en effet les soldats britanniques comme des jeunes gens qui, à cause du stress généré par leur mission en Irlande du Nord souffrent d'alopécie. Le prestige de leur fonction s'en trouve ainsi amoindri ; les soldats semblent terrorisés et leur apparence physique est risible. La satire est cependant loin de viser les seules troupes britanniques et les paramilitaires de l'IRA, comme les paramilitaires loyalistes ne sont pas épargnés par les réalisateurs. Barry McEvoy, qui (tout comme Barry Levinson) tenait à ce que le film soit tourné à Belfast, raconte d'ailleurs les problèmes que l'équipe a rencontrés auprès des membres de l'UDA, manifestement tout aussi mécontents du scénario :

The UDA had read the script and they didn't like it. The Northern Ireland Film Commission had been asking me to try and talk the studio into shooting there. They were sending me pictures and booklets, but they called the night before we were supposed to start, to say that negotiations with the UDA had broken down and they couldn't guarantee our safety. [...] Despite the real threat of sabotage, the shoot went ahead - though not without incident. One of the crew was hit by a bottle thrown over the peace wall ${ }^{29}$.

Tout comme Divorcing Jack, An Everlasting Piece met la ville de Belfast à l'écran de façon novatrice, et a recours à la satire pour représenter les groupes paramilitaires. An Everlasting Piece, qui date de 2000, aborde lui aussi la période des Troubles des années 1980 au travers du prisme de la comédie et les met en perspective en prenant également en compte l'évolution du processus de paix. Mais contrairement à Divorcing Jack, la conclusion du film est plutôt optimiste et fournit un espace privilégié où entrevoir la paix, comme le suggère le jeu de mots du titre An Everlasting Piece. Il s'agit en effet dans ce film de passer d'une société nord-irlandaise caractérisée par les divisions ethniques et religieuses de la période des Troubles à un avenir proche orienté vers la réconciliation.

Cette comédie se différencie des autres films sur les Troubles en ce qu'elle fait le choix de ne pas cristalliser à l'écran les images attendues d'une Irlande du Nord meurtrie par le conflit et se départit des contraintes de dates, spécifiant seulement que le film se passe à «Belfast à un certain moment des années $80^{30}$ ». Ironiquement, le début du film est situé dans un hôpital psychiatrique, microcosme symbolique où les relations entre catholiques et protestants sont le reflet des relations à l'échelle de la société nord-irlandaise. Colm O'Neill (Barry McEvoy), un jeune catholique, vient de trouver un emploi de coiffeur dans cette institution médicale. Bronagh (Anna Friel), sa petite amie catholique qui y travaille comme infirmière, lui explique qu'il n'y a que « cinq catholiques qui y travaillent, et que les patients sont presque tous catholiques » alors que les médecins, ainsi que ceux qui 
assurent la gestion et la direction de l'hôpital sont protestants. Le recours à l'image de l'établissement psychiatrique suggère l'idée que les catholiques ont tellement souffert qu'ils en auraient perdu la raison, et que de surcroît, ils demeurent opprimés et dominés par les protestants. C'est bien en fait la ville de Belfast qui est caricaturée par le biais de la métaphore de l'asile psychiatrique.

Le film montre comment le jeune Colm va s'employer à briser ce mode de fonctionnement sociétal qui obéit à des mœurs archaïques, elles-mêmes fondées sur une injustice sociale privilégiant les protestants. Les repères culturels des communautés catholique et protestante sont donc explorés à travers la dérision. Le pouvoir suprême des protestants, qui occupent tous les bureaux où se prennent les décisions de l'institut psychiatrique, est marqué par le comique de répétition tant dans le dialogue qu'à l'image. Ainsi, lorsque Colm est accueilli à l'hôpital pour la première fois, le directeur, Billy King (George Shane) lui présente tous ses collègues protestants : «We're all Billy. Billy Jewel, Billy Weatherly, Billy Evans, Billy Wilson, Billy William. I'm Billy King. They call me King Billy. No offence.» Alors que les portraits en plan moyen de chacun de ces hommes défilent les uns après les autres, le montage en parallèle (par ses effets d'alternance) renvoie à une image parodiée de la tradition du défilé orangiste. Et alors que tous les Billy se suivent avec leur cravate orange, leur lampe de bureau orange, leur jeu de cartes oranges ou encore leur classeur orange, les murs de l'hôpital peints en vert, blanc et orange, rappellent explicitement le drapeau tricolore irlandais, marqueur identitaire républicain. La dimension ironique et burlesque à l'image de la division sectaire s'impose, remettant en question le sectarisme et permettant de traiter le thème de la réconciliation.

17 C'est ainsi que Colm, le catholique, sympathise avec son collègue protestant George (Brian F. O'Byrne), un autre coiffeur, jeune homme sensible et amateur de poésie, ce qui rompt avec le cliché du protestant unioniste inculte :

Traditionnellement, les unionistes se sont toujours méfiés de la culture littéraire pas seulement à cause du calvinisme, de l'éthique du commerce, et de la peur de la liberté d'expression [...] mais parce qu'elle pouvait être «irlandaise » de manière latente. La Renaissance de la littérature irlandaise demeure nationaliste dans la mémoire des unionistes, quoi qu'en disent ceux qui critiquent aujourd'hui son « colonialisme ${ }^{31}$.

18 Ainsi, en offrant un portrait inédit du protestant, An Everlasting Piece illustre l'idée de réconciliation et s'inscrit dans la trajectoire du processus de paix. Au-delà de l'amitié qui se noue entre Colm et George, les deux hommes démarrent une relation professionnelle en créant ensemble leur propre entreprise. Tous deux découvrent que l'un des malades, un Ecossais catholique répondant au surnom de «Scalper » (Billy Connolly), a été interné pour avoir scalpé quatre de ses clients. Cet ancien homme d'affaires tenait un commerce de perruques et détenait le monopole du marché en Irlande du Nord. Colm et George, désireux de reprendre le commerce, réussissent à obtenir de «scalper" la liste des hommes chauves d'Ulster, qu'il conservait secrètement dans sa Bible. C'est ainsi que les deux coiffeurs, catholique et protestant, se lancent dans un commerce œcuménique qu'ils baptisent Piece People. Comme Colm l'explique à sa famille, les O'Neill, dont la maison est d'ailleurs située sur la «ligne de la démarcation de la paix » (Peace line) matérialisée par un mur et non pas dans le quartier catholique de Belfast, il s'agit d'une entreprise intercommunautaire, gérée par des personnes «non sectaires, des pacifistes». Ils vendent « des cheveux $100 \%$ protestants » à des pasteurs, des cheveux de religieuses dont le couvent est situé à Rome à des catholiques de Belfast, et démarchent aussi bien dans les quartiers républicains que loyalistes. De ce fait, Colm et George instaurent à Belfast une 
activité commerciale dont l'enjeu économique se double d'un enjeu politique, dans la mesure où ce commerce non sectaire s'oriente vers la réconciliation des deux communautés. La réconciliation demeure toutefois partielle, car la provenance des cheveux fait débat. En effet, alors que leur allégeance interconfessionnelle leur permet de vendre leurs propres perruques dans les deux camps, ils le font cependant en exploitant plutôt qu'en défiant les attitudes sectaires.

On le voit ici, le thème de la réconciliation doit se lire comme une anticipation du processus de guérison au Nord et dans le même temps, la violence paramilitaire est tournée en dérision et le recours à l'ironie ou à la satire cimentent l'idée principale d'un film, clairement orienté vers le processus de paix. Comme dans Divorcing Jack, An Everlasting Piece parodie les paramilitaires des deux bords et c'est à travers les deux héros que l'on découvre l'IRA et l'UDA, dont les représentants sont au fond assez pathétiques, préoccupés par leur calvitie, menacés dans leur virilité et hermétiques à l'idée même du processus de paix. M. Black de l'UDA est présenté comme un vieux garçon refoulé, qui conformément à ses aspirations puritaines prétend s'abstenir de toute activité sexuelle, mais est trahi lors d'un quiproquo autour du jeu de mots " hairpiece» et "herpes », qui laisse transparaître qu'il a bel et bien vécu des expériences sexuelles. Gêné, M. Black finit par acheter la perruque, exprimant la satisfaction d'être plus attirant physiquement.

Si le film se concentre sur le personnage de Black le loyaliste, l'image de l'IRA, qui est véhiculée par un groupe d'hommes anonymes, n'échappe pas pour autant à la caricature, qui demeure un moyen d'identifier les individus, en exploitant à l'envi l'iconographie paramilitaire républicaine. On découvre ainsi un groupe d'hommes cagoulés s'avançant en rase campagne dans la pénombre de la nuit. Manifestement interrompus dans leur opération par l'arrivée de Colm et George, qui se dirigeaient vers la ferme d'un de leurs clients potentiels, le chef de l'IRA (Colum Convey) et son bras droit (David Pearse) déstabilisés, les arrêtent. Mais le scénario rompt en partie avec les images stéréotypées de la violence de l'IRA, puisque le chef du groupe se montre menaçant envers George, non pas parce qu'il est protestant, mais plutôt parce qu'il le croit homosexuel. Le paramilitaire se révèle homophobe et, pensant surprendre les deux représentants en train de s'embrasser, ses hommes leur tendent une embuscade, et les font sortir violemment de leur véhicule avant de l'inspecter. La situation vire au burlesque lorsque, contre toute attente, le chef de l'IRA témoigne un grand intérêt pour les perruques découvertes dans le coffre, puis en essaie une, qu'il troque contre sa cagoule, et dont il souhaite faire l'acquisition. La scène burlesque se poursuit au-delà de cette image insolite: lorsque le chef s'aperçoit qu'il lui manque une livre pour payer la perruque, George et Colm se retrouvent dans une position de domination inattendue, et les rôles sont inversés. Le terroriste redevient un homme ordinaire, qui doit se soumettre aux règles du commerce, et est dépourvu de son aura de leader.

21 Qu'il s'agisse de l'UDA ou de l'IRA, le film opte pour une narration qui souligne le ridicule et le non-sens de l'action terroriste. An Everlasting Piece ridiculise tout aussi bien l'armée britannique que l'IRA, les loyalistes, et la société nord-irlandaise divisée par le sectarisme à travers une comédie qui, comme Northrop Frye le rappelle, « représente un mouvement de passage d'une certaine forme de société à une autre forme ${ }^{32}$ ». Dans le cas de An Everlasting Piece, il s'agit de passer de l'ancienne société nord-irlandaise, caractérisée par les questions ethniques et religieuses qui divisent les catholiques et les protestants pendant la période des Troubles, à une société réconciliée, afin d'aboutir à «la paix éternelle» (an everlasting peace) comme le jeu de mots du titre du film le sous-entend. 
C'est dans cette optique que Barry McEvoy a conçu son récit filmique. Selon lui, le choix de la comédie permet de se différencier des autres films sur les Troubles et des habituels clichés sur la violence au Nord :

\begin{abstract}
At one point we met an English director [...] to see about him making it, and he said, "I think we need a scene in here where the IRA are kneecapping someone". And I said, [...] If we're going to do that, we're going to need a scene where the SAS are torturing someone, and where the UDA pick up a random Catholic and kill him. And where do we stop? This isn't a movie about rubbing salt in wounds. I wanted to make a different film about Northern Ireland. I'm not saying it doesn't happen, but just not in this particular film ${ }^{33}$.
\end{abstract}

An Everlasting Piece s'inscrit donc sans ambiguïté dans l'optique du processus de paix : « The idea of the film is that you can't demonise the other side. [...] I don't think it helps Northern Ireland to demonise the other side ${ }^{34}$.» McEvoy rejette, entre autres, le « mythe de l'atavisme ${ }^{35}$ » de la violence nord-irlandaise et les images qui y sont associées, pour souligner que la comédie, utilisée comme structure narrative pour aborder les Troubles «pourrait jouer un rôle modeste dans le processus de guérison au Nord ${ }^{36}$ ». Pour autant, il semble bien que le film représente la communauté catholique sous un jour plus flatteur que la communauté protestante. George, qui est doté d'une sensibilité que l'on ne retrouve pas chez les autres protestants, de même qu'il est le seul à avoir de l'empathie à l'égard des catholiques, sort du lot. Mais son identité protestante unioniste est ambiguë. En effet, le personnage, qui est attiré par la culture littéraire irlandaise puisqu'il écrit et récite ses propres poèmes à l'Université de Queen's à Belfast, présente finalement une facette plus nationaliste et "irlandaise » qu'unioniste ou loyaliste, ce qui revient à définir George comme un «bon» protestant dans la mesure où il ressemble à un catholique. Sa disposition «naturelle » pour s'intégrer à la famille catholique de Colm, les O'Neill, est évidemment politiquement symbolique. Fidèle à la logique englobante de la comédie, le film montre protestants et catholiques réconciliés, et le fait que George soit en partie catholique participe de cela : «La comédie tend à rassembler le plus grand nombre possible de ses personnages dans la société instaurée par son dénouement. Au lieu d'être écartés, les opposants sont le plus souvent réconciliés ou convertis ${ }^{37}$." C'est donc particulièrement dans le dénouement (qui prône la paix en Irlande du Nord) que le discours biaisé du film apparaît le plus clairement, car An Everlasting Piece démontre que les catholiques sont en fait les plus actifs et les plus volontaires dans le processus de paix. Si les Piece People refusent de vendre des perruques aux membres de l'IRA pour des questions éthiques, ils organisent une vente importante auprès de l'armée britannique, dont les soldats souffrent d'alopécie, ce que l'on peut interpréter comme une manière de montrer que la communauté nord-irlandaise rompt avec l'IRA et qu'elle prend aussi l'initiative d'aller vers les Britanniques. Mais la raison pour laquelle Colm consent à mener à bien la vente de perruques à l'armée britannique reste morale, et il conçoit cet acte en se référant aux valeurs de la religion catholique ( $a$ gesture »). À travers ce personnage, An Everlasting Piece présente la communauté catholique comme seule victime $\mathrm{du}$ gouvernement britannique et donc comme unique vecteur du "pardon». L élan commun des deux héros Colm et George vers la réconciliation ne semble qu'anecdotique, puisque c'est au final Colm, le héros catholique, qui s'octroie le pouvoir exclusif de gérer 
et de résoudre le conflit. Il n'en ressort pas moins que tout " geste » représente toujours une étape vers la paix, et que An Everlasting Piece se positionne en faveur de ce processus.

Divorcing Jack et An Everlasting Piece s'intègrent donc dans la mouvance du processus de paix et ajoutent de nouvelles représentations à la palette cinématographique du conflit politique nord-irlandais, abordé cette fois à travers la comédie. Il s'avère en effet que le processus de paix autorise une exploration sans tabous de la violence politique, qui est non seulement dénoncée mais choisie comme objet de la comédie. Toutefois, il faut souligner que les discours filmiques divergent quant à l'avenir de l'Irlande du Nord. Alors que Divorcing Jack se projette dans la période qui suit le processus de paix et opte pour le scénario d'une l'Irlande du Nord indépendante, le film demeure pessimiste, évoquant la perpétuation de la violence par les politiciens corrompus et les paramilitaires Malgré sa position assez ouvertement pro-catholique, An Everlasting Piece véhicule une vision plus optimiste et suggère la possibilité d'une réconciliation. Il convient aussi de noter la particularité de ces deux films, qui demeurent les seules depuis 2001 à avoir fait le choix de la comédie pour évoquer les Troubles ou le processus de paix. Ces deux périodes continuent d'être explorées à l'écran comme dans les films Omagh de Pete Travis (2004) et Perrier's Bounty de Ian Fizgibbon (2009. The Mighty Celt de Pearse Elliot (2005) et Breakfast on Pluto de Neil Jordan (2005) qui, contiennent quelques éléments comiques, utilisent les Troubles seulement comme toile de fond et n'articulent pas l'intrigue autour de ce sujet. Ce constat donne à penser que, même si la période contemporaine à la signature de l'Accord du Vendredi Saint avait permis d'espérer et de projeter ces espoirs à l'écran, rire de la violence politique en Irlande du Nord reste une question délicate plus de dix ans après cet événement.

\section{NOTES}

1. Hennessy de Don Sharp (1975), The Long Good Friday de John Mc Kenzie (1979), A Prayer for the Dying de Mike Hodges (1987) et Hidden Agenda de Ken Loach (1990) n'explorent pas précisément la situation politique en Irlande du Nord puisque d'une part ils explorent ses répercussions en Angleterre, et d'autre part, ils véhiculent l'image de l'IRA à travers des individus isolés de la société ou encore des groupes non identifiés, invisibles à l'écran. Ces films contribuent à noircir le conflit politique nord-irlandais, présenté comme incompréhensible et renforcent les représentations stéréotypées relatives à cette situation. Seule l'idéologie républicaine est représentée, ce qui engendre une vision relativement simpliste et caricaturale, voire manichéenne, du conflit. Angel de Neil Jordan (1982) et Cal de Pat O'Connor (1983), réalisés par des cinéastes irlandais, explorent la violence politique exclusivement en Ulster, et par conséquent donnent une autre dimension au conflit. Même s'il est impossible de distinguer clairement les identités paramilitaires (dans Angel), elles sont néanmoins suggérées, et le film n'est pas dépourvu d'implications politiques puisqu'il finit par dénoncer implicitement la collusion entre la RUC et les paramilitaires loyalistes. Cal dénonce clairement la violence paramilitaire en Irlande du Nord et illustre la violence sectaire dont la communauté catholique est 
montrée comme la principale victime. Le film distingue toutefois les loyalistes du reste des protestants. Maeve, de la réalisatrice nord-irlandaise Pat Murphy (1981), explore les Troubles à travers le regard féministe qu'une femme porte sur la communauté catholique et l'IRA en s'exilant à Londres, retournant à Belfast seulement pour rendre visite à sa famille. Le point de vue féministe de Maeve dénonce l'image de la femme irlandaise passive et soumise à l'autorité masculine et le film tente de démontrer que les mythes fondateurs républicains et la culture nationaliste tiennent la femme à l'écart du discours officiel de l'histoire de l'Irlande et du conflit politique nord-irlandais.

2. On observe une dynamique pacifiste dans la mesure où ces films présentent, pour la plupart, des héros qui ne sont pas dans l'IRA ou qui tentent de s'en écarter. Inspirés de l'approche post-coloniale, les films The Crying Game de Neil Jordan (1992) et High Boot Benny de Joe Comerford (1993) placent au coeur de leur structure narrative le problème identitaire et remettent en question les stéréotypes du conflit politique nord-irlandais. Some Mother's Son de Terry George (1996) et $\mathrm{H} 3$ de Les Blair (2001) traitent des prisonniers républicains et des grèves de la faim. Le premier film aborde le sujet du point de vue d'une mère qui condamne la violence politique alors que le deuxième sacralise à l'écran la mémoire collective des prisonniers républicains. Le film Silent Grace de Maeve Murphy (2001) aborde également le sujet des grèves de la faim, mais à travers le personnage d'une femme militante activiste de l'IRA et prisonnière politique dans la prison d'Armagh. In the Name of the Father de Jim Sheridan (1993) représente le scandale judiciaire des « Quatre de Guilford ». Quant à The Boxer de Jim Sheridan (1997), il explore le parcours d'un boxeur nord-irlandais qui tente de redonner un sens à sa vie en s'émancipant de l'IRA, en luttant notamment contre la violence à Belfast. Les positions divergentes relatives au cessez-lefeu entre les membres de l'IRA y sont soulignées. Titanic Town de Roger Mitchell (1998) est un film qui met en scène une héroïne, qui de mère catholique devient médiateur de paix, engagée contre la violence politique qui menace sa famille au quotidien. Bloody Sunday de Paul Greengrass (2001) et Sunday de Charles McDougal (2001) retracent à l'écran une tragédie de l'histoire des Troubles, celle du Dimanche sanglant de 1969. Enfin, les films Nothing Personal de Thaddeus O'Sullivan (1995), Resurrection Man de Marc Evans (1998) et As the Beast Sleeps de Harry Bradbeer (2001) observent le conflit politique nord-irlandais à travers les images des protestants, particulièrement des loyalistes.

3. On note toutefois une différence entre le film Divorcing Jack où la violence paramilitaire est parodiée et le film An Everlasting Piece où la violence paramilitaire est suggérée, intervenant ainsi dans le hors champ, non visible à l'écran.

4. Marc Ferro, Cinéma et Histoire, Paris, Gallimard, 1993.

5. Ibid, p. 214.

6. Angel de Neil Jordan (1982), High Boot Benny de Joe Comerford (1993), Nothing Personal de Thaddeus O'Sullivan (1995), Some Mother's Son de Terry George (1996), Resurrection Man de Marc Evans (1998) par exemple.

7. Colin Bateman, Divorcing Jack, London, Harper Collins, 1994.

8. M. Heaney, «Intelligence from the Y-Front », Sunday Times, 28/11/99, p. 18.

9. «I'm not political or religious in any way », Ibid. p. 18.

10. Ibid, p. 18.

11. Si le film suggère que l'histoire se passe après l'Accord du Vendredi Saint, Divorcing Jack n'explore pas les institutions prévues par ce texte, mais imagine l'Irlande du Nord indépendante. 
12. David Caffrey, " "The Only Way is Up", An Interview with David Caffrey by Joanne Haden » disponible [www.iol.ie/galfilm/filmwest].

13. "The humour is so dangerously on the edge - like making jokes about the Holocaust - that at times you're almost afraid to laugh ", Sunday Tribune, " Nun's the word as "Divorcing Jack" says the unspeakable », 18/10/98, p. 6 .

14. Pierre Sorlin, "Rire de l'horreur? Le comique de guerre", in Cinéma, le genre comique, Université Paul Valéry-Montpellier 3, 1997, p. 163.

15. Ibid. p. 159.

16. Martin McLoone, Irish Film: The Emergence of a Contemporary Cinema, London, BFI, 2000, p. 83.

17. Pierre Sorlin observe que dans ce genre de comédies, «les anti-héros attirent l'indulgence par leur faiblesse avouée ». Ibid. p. 161.

18. John Hill, Cinema and Northern Ireland, London, British Film Institute, 2006, p. 213.

19. Pierre Sorlin, op. cit., p. 156.

20. Avant An Everlasting Piece, Barry Levinson réalisa entre autres Good Morning Vietnam (1987), Rain Man (1988) qui obtint l'Oscar du meilleur réalisateur en 1989 ainsi que le César du meilleur film étranger en 1990, Avalon (1990), Bugsy (1991), Disclosure (1994), Sleepers (1996), Wag The Dog (1997), Liberty Heights (1999).

21. Steven Spielberg est l'un des trois directeurs de cette maison de production.

22. The Irish Times, 19/03/01, p. 9. Les auteurs ont d'ailleurs exprimé leur mécontentement dans plusieurs entretiens, notamment Barry Levinson dans The Guardian (Friday Review), 23/03/01. p. 10.

23. Se référer à l'article de Michael Gray, Film West, Spring 2001, n 43, p. 19.

24. "[...] on precisely eight screens. They ran it for a week, then told us it wasn't working and closed it". The Guardian, 23/03/01, p. 10.

25. "He said: 'Cut the politics', Barry said :'No' and we were released on eight screens." The Irish Times 19/03/01, p. 9.

26. Jerome O'Connor a perdu ce procès.

27. "[...] it buried the film at the behest of the British government", in "The Politics of Piece", Sunday Tribune, 25/02/01, p. 3.

28. "The Politics of Piece", Sunday Tribune, 25/02/01, p. 3.

29. Michael Gray, « An Everlasting Piece. Barry McEvoy Interview », Film West, Spring 2001, $\mathrm{n}^{\circ} 43$, p. 19.

30. «Belfast, sometime during the 80's. »

31. Edna Longley, «Opening up: A New Pluralism », Fortnight, n² 256, novembre 1987, p.

24. Traduit par Wesley Hutchinson, Espaces de l'imaginaire unioniste nord-irlandais, Caen, Presses Universitaires de Caen, 1999, p. 43.

32. Northrop Frye, Anatomie de la critique (traduit de l'anglais par Guy Durand, Anatomy of Criticism. 1957), Paris, Editions Gallimard, 1969, p. 199.

33. Michael Gray, « An Everlasting Piece: Barry McEvoy Interview », Film West, Spring 2001, $n^{\circ} 43$, p. 18.

34. Ibid., p. 19. 
35. Comme l'observe Martin McLoone, «le mythe de l'atavisme» est un thème prédominant dans les films traitant des Troubles réalisés pendant la période des Troubles comme dans Hennessy ou encore The Long Good Friday. Irish Film, The Emergence of a Contemporary Cinema, London, British Film Institute, 2000, p. 60-85.

36. «[...] might have a modest part to play in the North's healing process », Ibid., p. 19.

37. Northrop Frye, op. cit., p. 201.

\section{RÉSUMÉS}

Les films sur le conflit politique en Irlande du Nord, assez répandus depuis ces deux dernières décennies, reflètent les changements qui se sont produits en Irlande du Nord. Les comédies qui ont commencé à apparaitre à l'écran à partir de l'Accord du Vendredi Saint ne font pas exception et observent ce changement, offrant un véhicule unique pour aborder les traumatismes du passé de l'Irlande du Nord. Cet article qui traite de deux comédies sur les Troubles et le processus de paix, Divorcing Jack (1998) et An Everlasting Piece (2000), analyse les façons dont elles explorent le changement historique, culturel et politique en Irlande du Nord, à travers l'ironie, et aussi, comment elles fournissent un espace pour de nouvelles interprétations des Troubles et du processus de paix.

There has been a growing number of films about the political conflict in Northern Ireland in the last two decades, all of which have reflected changes in Northern Ireland. Similarly, the comedy films which started emerging around the time of the Good Friday Agreement have accounted for these changes and offered a unique medium to adress Northern Ireland's traumatic past. This article, which deals with two comedy films about the Troubles and the Peace process, Divorcing Jack (1998) and An Everlasting Piece (2000), analyses the ways in which they explore historical, cultural and political change in Northern Ireland through irony, and also how they provide a space for new interpretations of the Troubles and the peace process.

\section{INDEX}

Mots-clés : cinéma, Irlande du Nord - paramilitaires, Irlande du Nord - post-conflit, Irlande du Nord - processus de paix, histoire des représentations, Irlande du Nord - conflit

Keywords : cinema, Northern Ireland - paramilitaries, Northern Ireland - peace process, Northern Ireland - post-conflict, history of representations, Northern Ireland - conflict

\section{AUTEUR}

\section{CÉCILE BAZIN}

Université Paris III-Sorbonne Nouvelle 